\title{
Diagnosis, Evaluation, Prevention, and Treatment of Chronic Kidney Disease-Mineral and Bone Disorder: Synopsis of the Kidney Disease: Improving Global Outcomes 2017 Clinical Practice Guideline Update
}

\author{
Markus Ketteler, MD; Geoffrey A. Block, MD; Pieter Evenepoel, MD, PhD; Masafumi Fukagawa, MD, PhD; Charles A. Herzog, MD; \\ Linda McCann, RD, CSR; Sharon M. Moe, MD; Rukshana Shroff, MD, PhD; Marcello A. Tonelli, MD, SM, MSc; \\ Nigel D. Toussaint, MBBS, PhD; Marc G. Vervloet, MD, PhD; and Mary B. Leonard, MD, MSCE
}

Description: The Kidney Disease: Improving Global Outcomes (KDIGO) 2017 Clinical Practice Guideline Update for the Diagnosis, Evaluation, Prevention, and Treatment of Chronic Kidney Disease-Mineral and Bone Disorder (CKD-MBD) is a selective update of the prior CKD-MBD guideline published in 2009. The guideline update and the original publication are intended to assist practitioners caring for adults with CKD and those receiving long-term dialysis.

Methods: Development of the guideline update followed an explicit process of evidence review and appraisal. The approach adopted by the Work Group and the evidence review team was based on systematic reviews of relevant trials, appraisal of the quality of the evidence, and rating of the strength of recommendations according to the GRADE (Grading of Recommendations Assessment, Development and Evaluation) approach. Searches of the English-language literature were conducted through September 2015 and were supplemented with targeted searches through February 2017. Final modification of the guidelines was informed by a public review process involving numerous stakeholders, including patients, subject matter experts, and industry and national organizations.

Recommendations: The update process resulted in the revision of 15 recommendations. This synopsis focuses primarily on recommendations for diagnosis of and testing for CKD-MBD and treatment of CKD-MBD that emphasizes decreasing phosphate levels, maintaining calcium levels, and addressing elevated parathyroid hormone levels in adults with CKD stage G3a to $G 5$ and those receiving dialysis. Key elements include basing treatment on trends in laboratory values rather than a single abnormal result and being cautious to avoid hypercalcemia when treating secondary hyperparathyroidism.

Ann Intern Med. doi:10.7326/M17-2640

Annals.org

For author affiliations, see end of text.

This article was published at Annals.org on 20 February 2018.
$\mathbf{C}^{\mathrm{h}}$ hronic kidney disease (CKD) is defined as abnormalities in kidney structure or function that are present for more than 3 months and have health implications. The disease is classified on the basis of cause and category of glomerular filtration rate (GFR) (G1 to G5) and albuminuria (A1 to A3) (Appendix Figure, available at Annals.org). As kidney function decreases, marked changes in bone mineral metabolism occur, resulting in increased risk for fractures, cardiovascular disease, and overall mortality. In 2009, Kidney Disease: Improving Global Outcomes (KDIGO) published the Clinical Practice Guideline for the Diagnosis, Evaluation, Prevention, and Treatment of Chronic Kidney Disease-Mineral and Bone Disorder (CKD-MBD) (1). Based on evidence from new clinical trials, an updated clinical practice guideline was published in 2017 (2).

The 2017 update (available at www.kdigo.org) provides recommendations for diagnosis of bone abnormalities in CKD-MBD, treatment of CKD-MBD by decreasing serum phosphate levels and maintaining serum calcium levels, treatment of parathyroid hormone (PTH) abnormalities in CKD-MBD, treatment of bone abnormalities using antiresorptive agents and other osteoporosis therapies, and evaluation and treatment of kidney transplant bone disease (2). This synopsis focuses on diagnosis of CKD-MBD and management of serum phosphate, calcium, and PTH levels in adults-areas in which controversy and knowledge gaps exist. Recommendations for children and kidney transplant recipients are not addressed in this synopsis, but interested readers can refer to the guideline update for details (2).

A consolidated listing of CKD-MBD guideline statements relevant to adults with CKD stage G3a to G5 and those receiving dialysis, including the revised recommendations in the 2017 guideline update, is provided in the Table. The target audience for the guideline includes nephrologists, primary care physicians, and other health professionals caring for adults with CKD or those receiving dialysis.

\section{Guideline Development Process, Evidence Grading, and Stakeholder and Public \\ Consultation}

The KDIGO Controversies Conference, held in October 2013, determined that there was sufficient new evidence to support updating some of the CKDMBD recommendations (3). The guideline update process began with the formation of an international Work Group and an independent evidence review team

\author{
See also: \\ Web-Only \\ $\mathrm{CME} / \mathrm{MOC}$ activity
}


Table. Consolidated KDIGO Guideline Recommendations for Adults With CKD Stage G3a to G5D and CKD-MBD*

Chapter 3.1: Diagnosis of CKD-MBD: Biochemical Abnormalities

3.1.1: We recommend monitoring serum levels of calcium, phosphate, PTH, and alkaline phosphatase activity beginning in CKD G3a. (Grade $1 \mathrm{C}$ recommendation)

3.1.2: In patients with CKD G3a to G5D, it is reasonable to base the frequency of monitoring serum calcium, phosphate, and PTH on the presence and magnitude of abnormalities, and the rate of progression of CKD. (Not graded)

Reasonable monitoring intervals would be:

- In CKD G3a to G3b: for serum calcium and phosphate, every 6-12 months; and for PTH, based on baseline level and CKD progression

- In CKD G4: for serum calcium and phosphate, every 3-6 months; and for PTH, every 6-12 months

- In CKD G5, including G5D: for serum calcium and phosphate, every 1-3 months; and for PTH, every 3-6 months

- In CKD G4 to G5D: for alkaline phosphatase activity, every 12 months, or more frequently in the presence of elevated PTH (see Chapter 3.2)

In CKD patients receiving treatments for CKD-MBD, or in whom biochemical abnormalities are identified, it is reasonable to increase the frequency of measurements to monitor for trends and treatment efficacy and side effects. (Not graded)

3.1.3: In patients with CKD G3a to G5D, we suggest that 25-(OH)D (calcidiol) levels might be measured, and repeated testing determined by baseline values and therapeutic interventions. (Grade 2C recommendation) We suggest that vitamin $D$ deficiency and insufficiency be corrected using treatment strategies recommended for the general population. (Grade $2 \mathrm{C}$ recommendation)

3.1.4: In patients with CKD G3a to G5D, we recommend that therapeutic decisions be based on trends rather than on a single laboratory value, taking into account all available CKD-MBD assessments. (Grade 1C recommendation)

3.1.5: In patients with CKD G3a to G5D, we suggest that individual values of serum calcium and phosphate, evaluated together, be used to guide clinical practice rather than the mathematical construct of calcium-phosphate product ( $\mathrm{Ca} \times \mathrm{P})$. (Grade 2D recommendation)

3.1.6: In reports of laboratory tests for patients with CKD G3a to G5D, we recommend that clinical laboratories inform clinicians of the actual assay method in use and report any change in methods, sample source (plasma or serum), and handling specifications to facilitate the appropriate interpretation of biochemistry data. (Grade 1B recommendation)

Chapter 3.2: Diagnosis of CKD-MBD: Bone Abnormalities 3.2.1: In patients with CKD G3a to G5D with evidence of CKD-MBD and/or risk factors for osteoporosis, we suggest BMD testing to assess fracture risk if results will impact treatment decisions. (Grade 2B recommendation)

3.2.2: In patients with CKD G3a to G5D, it is reasonable to perform a bone biopsy if knowledge of the type of renal osteodystrophy will impact treatment decisions. (Not graded)

3.2.3: In patients with CKD G3a to G5D, we suggest that measurements of serum PTH or bone-specific alkaline phosphatase can be used to evaluate bone disease because markedly high or low values predict underlying bone turnover. (Grade 2B recommendation)

3.2.4: In patients with CKD G3a to G5D, we suggest not to routinely measure bone-derived turnover markers of collagen synthesis (such as procollagen type I C-terminal propeptide) and breakdown (such as type I collagen cross-linked telopeptide, cross-laps, pyridinoline, or deoxypyridinoline). (Grade 2C recommendation)

Chapter 3.3: Diagnosis of CKD-MBD: Vascular Calcification

3.3.1: In patients with CKD G3a to G5D, we suggest that a lateral abdominal radiograph can be used to detect the presence or absence of vascular calcification, and an echocardiogram can be used to detect the presence or absence of valvular calcification, as reasonable alternatives to computed tomography-based imaging. (Grade 2C recommendation)

Continued
Table-Continued

3.3.2: We suggest that patients with CKD G3a to G5D with known vascular or valvular calcification be considered at highest cardiovascular risk. (Grade 2A recommendation) It is reasonable to use this information to guide the management of CKD-MBD. (Not graded)

Chapter 4.1: Treatment of CKD-MBD Targeted at Lowering High Serum Phosphate and Maintaining Serum Calcium

4.1.1: In patients with CKD G3a to G5D, treatments of CKD-MBD should be based on serial assessments of phosphate, calcium, and PTH levels, considered together. (Not graded)

4.1.2: In patients with CKD G3a to G5D, we suggest lowering elevated phosphate levels toward the normal range. (Grade 2C recommendation)

4.1.3: In adult patients with CKD G3a to G5D, we suggest avoiding hypercalcemia. (Grade $2 C$ recommendation)

4.1.4: In patients with CKD G5D, we suggest using a dialysate calcium concentration between 1.25 and $1.50 \mathrm{mmol} / \mathrm{L}$ (2.5 and 3.0 $\mathrm{mEq} / \mathrm{L}$ ). (Grade $2 \mathrm{C}$ recommendation)

4.1.5: In patients with CKD G3a to G5D, decisions about phosphate-lowering treatment should be based on progressively or persistently elevated serum phosphate. (Not graded)

4.1.6: In adult patients with CKD G3a to G5D receiving phosphate-lowering treatment, we suggest restricting the dose of calcium-based phosphate binders. (Grade $2 B$ recommendation)

4.1.7: In patients with CKD G3a to G5D, we recommend avoiding the long-term use of aluminum-containing phosphate binders and, in patients with CKD G5D, avoiding dialysate aluminum contamination to prevent aluminum intoxication. (Grade $1 \mathrm{C}$ recommendation)

4.1.8: In patients with CKD G3a to G5D, we suggest limiting dietary phosphate intake in the treatment of hyperphosphatemia alone or in combination with other treatments. (Grade $2 \mathrm{D}$ recommendation) It is reasonable to consider phosphate source (e.g., animal, vegetable, additives) in making dietary recommendations. (Not graded)

4.1.9: In patients with CKD G5D, we suggest increasing dialytic phosphate removal in the treatment of persistent hyperphosphatemia. (Grade $2 \mathrm{C}$ recommendation)

Chapter 4.2: Treatment of Abnormal PTH Levels in CKD-MBD 4.2.1: In patients with CKD G3a to G5 not on dialysis, the optimal PTH level is not known. However, we suggest that patients with levels of intact PTH progressively rising or persistently above the upper normal limit for the assay be evaluated for modifiable factors, including hyperphosphatemia, hypocalcemia, high phosphate intake, and vitamin D deficiency. (Grade 2C recommendation)

4.2.2: In adult patients with CKD G3a to G5 not on dialysis, we suggest that calcitriol and vitamin $D$ analogues not be routinely used. (Grade $2 \mathrm{C}$ recommendation) It is reasonable to reserve the use of calcitriol and vitamin D analogues for patients with CKD G4 to G5 with severe and progressive hyperparathyroidism. (Not graded)

4.2.3: In patients with CKD G5D, we suggest maintaining iPTH levels in the range of approximately two to nine times the upper normal limit for the assay. (Grade $2 \mathrm{C}$ recommendation)

We suggest that marked changes in PTH levels in either direction within this range prompt an initiation or change in therapy to avoid progression to levels outside of this range. (Grade 2C recommendation)

4.2.4: In patients with CKD G5D requiring PTH-lowering therapy, we suggest calcimimetics, calcitriol, or vitamin $D$ analogues, or a combination of calcimimetics with calcitriol or vitamin $D$ analogues. (Grade $2 B$ recommendation)

4.2.5: In patients with CKD G3a to G5D with severe hyperparathyroidism (HPT) who fail to respond to medical or pharmacological therapy, we suggest parathyroidectomy. (Grade 2B recommendation)

Chapter 4.3: Treatment of Bone With Bisphosphonates, Other Osteoporosis Medications, and Growth Hormone

4.3.2: In patients with CKD G3a to G3b with PTH in the normal range and osteoporosis and/or high risk of fracture, as identified by World Health Organization criteria, we suggest treatment as for the general population. (Grade 2B recommendation)

Continued on following page 


\begin{tabular}{l}
\hline Table-Continued \\
\hline 4.3.3: In patients with CKD G3a to G5D with biochemical \\
abnormalities of CKD-MBD and low BMD and/or fragility fractures, \\
we suggest that treatment choices take into account the magnitude \\
and reversibility of the biochemical abnormalities and the \\
progression of CKD, with consideration of a bone biopsy. (Grade \\
2D recommendation)
\end{tabular}

$25-(\mathrm{OH}) \mathrm{D}=25$-hydroxyvitamin $\mathrm{D} ; \mathrm{BMD}=$ bone mineral density; $\mathrm{CKD}=$ chronic kidney disease; $\mathrm{CKD}-\mathrm{MBD}=$ chronic kidney diseasemineral and bone disorder; $\mathrm{PTH}=$ intact parathyroid hormone; KDIGO = Kidney Disease: Improving Global Outcomes; PTH = parathyroid hormone.

* Chapters 1 and 2 of the 2009 CKD-MBD guideline (1) provide the introduction and methodological approach, respectively; therefore, guideline recommendations begin in chapter 3.1. Guideline statements pertaining to pediatric and kidney transplant recipient populations are not addressed in this synopsis but can be found in the guideline update (2). Updated statements are italicized and boldfaced.

based at Johns Hopkins University in Baltimore, Maryland.

As with the original 2009 KDIGO CKD-MBD guideline (1), the 2017 update process relied on rigorous review and appraisal of the evidence derived from systematic reviews of clinical trial results, using the GRADE (Grading of Recommendations Assessment, Development and Evaluation) approach (4) (Appendix Tables 1 and 2, available at Annals.org). Briefly, the process included refining the research questions, developing the literature search strategy, revising the 2009 recommendation statements, and grading evidence quality and the strength of recommendations (Appendix Table 3, available at Annals.org). Each recommendation was accompanied by the strength of the recommendation and an evidence grade. Guideline statements that provided general advice or guidance (and thus were not based on systematic review) were marked "not graded."

The guideline development process included an external public review to ensure widespread input from patients, experts, and industry and national organizations. Final revisions were reviewed and incorporated before publication of the guideline update.

\section{UPDATED RECOMMENDATIONS RELATING TO Diagnosis of Bone AbNoRmalities in CKD-MBD}

3.2.1: In patients with CKD G3a to G5D with evidence of CKD-MBD and/or risk factors for osteoporosis, we suggest $B M D$ testing to assess fracture risk if results will impact treatment decisions. (Grade 2B recommendation)

When the 2009 KDIGO CKD-MBD guideline was published, cross-sectional studies of dual-energy x-ray absorptiometry (DXA) that compared bone mineral density (BMD) in patients with CKD with and without a prevalent fracture were limited. Consequently, the 2009 guideline recommended that BMD testing not be routinely performed in patients with CKD stage G3a to G5D and CKD-MBD (1).

The evidence review for the 2017 KDIGO CKDMBD guideline update identified 4 prospective cohort studies in adults showing that DXA BMD testing pre- dicted fractures across the spectrum from CKD stage G3a to G5D (5-8). Although the studies were conducted across a range of CKD severity, the finding that hip BMD predicted fractures was consistent across studies. Two studies demonstrated associations similar to those seen in the absence of $\operatorname{CKD}(6,8)$.

The evidence review also examined results from 3 new clinical trials that studied the effects of osteoporosis medications on BMD in CKD stage G3a to G5D (911). However, the studies did not show consistent beneficial effects of osteoporosis medications on BMD.

In conclusion, DXA BMD assessment is reasonable if low or decreasing BMD will lead to additional interventions to reduce falls or recommendations for use of osteoporosis medications.

3.2.2: In patients with CKD G3a to G5D, it is reasonable to perform a bone biopsy if knowledge of the type of renal osteodystrophy will impact treatment decisions. (Not graded)

Bone biopsy is the gold standard for diagnosis and classification of renal osteodystrophy (12). The 2009 guideline noted that DXA BMD testing does not distinguish among types of renal osteodystrophy, and the diagnostic utility of biochemical markers was limited by their poor sensitivity and specificity (1).

A study of bone biopsies from 492 patients receiving dialysis (13) found that no biomarker (alone or in combination with others) was sufficiently robust to diagnose low, normal, and high bone turnover in individual patients. Differences in PTH assays have also contributed to conflicting results across studies.

Due to these considerations, therapeutic decisions should be based on trends in serum PTH levels instead of 1-time values. When PTH trends are inconsistent, it is reasonable to perform bone biopsy if the results could lead to changes in therapy.

The 2009 guideline recommended bone biopsy before antiresorptive therapy in patients with CKD stage G4 to G5D and evidence of biochemical abnormalities of CKD-MBD, low BMD, and/or fragility fractures (1). However, due to limited clinical experience with performance of bone biopsy and evaluation of the results (14), as well as growing evidence that antiresorptive therapies are effective in patients with CKD stage G3a to G4, bone biopsy is no longer a prerequisite for initiation of these therapies.

\section{UPDATED RECOMMENDATIONS RELATING TO Management of Serum Phosphate and Calcium Levels}

4.1.1: In patients with CKD G3a to G5D, treatments of CKD-MBD should be based on serial assessments of phosphate, calcium, and PTH levels, considered together. (Not graded)

4.1.2: In patients with CKD G3a to G5D, we suggest lowering elevated phosphate levels toward the normal range. (Grade $2 \mathrm{C}$ recommendation)

In patients with CKD, clinical decisions are routinely based on serum phosphate, calcium, and PTH concen- 
trations. However, these are influenced by several factors, including diurnal changes $(15,16)$. A recent post hoc analysis of large dialysis cohorts suggested that the prognostic implications of individual biochemical components of CKD-MBD largely depend on their context within the full array of MBD biomarkers (17). This analysis identified a wide range of CKD-MBD phenotypes, based on phosphate, calcium, and PTH measurements segregated into mutually exclusive categories (low, medium, and high) using previous targets from the KDIGO guideline as well as earlier Kidney Disease Outcomes Quality Initiative guidelines. The analysis underscored the importance of potential interactions among components of CKD-MBD in terms of risk prediction for death or cardiovascular events. Treatments aimed at improving one variable often have unintended or intended effects on others (18). Thus, treatment decisions should be based not on a single laboratory value but on trends of serial measurements of phosphate, calcium, and PTH considered together.

High-quality evidence now links high phosphate concentrations with mortality among patients with CKD stage G3a to G5 and transplant recipients (19-28). However, there is still a lack of data from clinical trials showing that therapeutic approaches to decreasing serum phosphate levels improve patient-centered outcomes.

Methods for preventing hyperphosphatemia include diet modification, phosphate-lowering therapy, and intensified dialysis for patients with CKD stage G5D. The 2009 guideline suggested maintenance of normal serum phosphate levels for patients with CKD stages G3a to G4.

Most studies found phosphate to be consistently associated with excess mortality at levels above and below the limits of normal but not in the normal range. However, a recent trial comparing placebo with active phosphate binder therapy in patients with CKD who were not receiving dialysis (stage G3b or G4) and who had normal phosphate concentrations before initiation of binder treatment found a minimal decrease in serum phosphate levels, no effect on fibroblast growth factor 23 (FGF23) levels, and increases in coronary calcification scores in the active treatment group (29). This led to concerns about the efficacy and safety of phosphate binders in this population.

On the basis of the current evidence, the previous suggestion to maintain normal phosphate levels was abandoned; instead, treatment should be focused on patients with hyperphosphatemia. Prevention rather than treatment of hyperphosphatemia may be valuable in patients with CKD stage G3a to G5D, but future studies will need to address the potential value of hyperphosphatemia prevention in at-risk CKD populations (for example, patients with elevated FGF23 levels).

4.1.3: In adult patients with CKD G3a to G5D, we suggest avoiding hypercalcemia. (Grade 2C recommendation)

4.1.4: In patients with CKD G5D, we suggest using a dialysate calcium concentration between 1.25 and $1.50 \mathrm{mmol} / \mathrm{L}(2.5$ and $3.0 \mathrm{mEq} / \mathrm{L}$ ). (Grade $2 \mathrm{C}$ recommendation)

Similar to phosphate, new data support an association between higher calcium concentrations and increased mortality in adults with CKD (22-24, 27, 30-34). Higher serum calcium concentrations have also been linked to nonfatal cardiovascular events $(35,36)$.

Hypocalcemia contributes to the pathogenesis of secondary hyperparathyroidism (SHPT) and renal osteodystrophy, prompting the 2009 recommendation to suggest maintenance of normal serum calcium levels, including correction of hypocalcemia. However, whether the suggestion to correct hypocalcemia was generalizable to all CKD stages and all treatment conditions is unclear on the basis of recent studies. One consideration is the potential harm associated with a positive calcium balance in some cases $(37,38)$. The second consideration is that the prevalence of hypocalcemia may have increased after the introduction of calcimimetics (cinacalcet) in patients receiving dialysis (18, $39,40)$. The clinical implications of this increased incidence are uncertain. On one hand, hypocalcemia represents the mode of action of calcimimetics and may positively contribute to bone mineralization. On the other hand, none of the pivotal trials or the phase 4 outcome trial EVOLVE (EValuation Of Cinacalcet Hydrochloride [HCl] Therapy to Lower CardioVascular Events) showed any adverse associations with mildly or moderately decreased calcium levels. The intention-to-treat analysis of the EVOLVE trial showed no association between negative signals and the persistently low serum calcium levels in the cinacalcet group (41).

The 2009 recommendation supported the concept that patients developing hypocalcemia during calcimimetic treatment require aggressive calcium treatment. Given the unproven benefits of calcimimetic treatment and the potential for harm, an individualized approach should be used to treat hypocalcemia rather than recommending correction of hypocalcemia in all patients. However, patients with significant or symptomatic hypocalcemia could still benefit from correction to prevent adverse consequences.

On the basis of new evidence $(42,43)$, the 2009 recommendation for dialysate calcium concentration was retained, but the evidence was upgraded from 2D to $2 \mathrm{C}$.

4.1.5: In patients with CKD G3a to G5D, decisions about phosphate-lowering treatment should be based on progressively or persistently elevated serum phosphate. (Not graded)

4.1.6: In adult patients with CKD G3a to G5D receiving phosphate-lowering treatment, we suggest restricting the dose of calcium-based phosphate binders. (Grade 2B recommendation)

New pathophysiologic understanding of phosphate regulation and the roles of FGF23 and soluble Klotho in early CKD have prompted studies investigating phosphate-lowering therapies in patients with CKD who have not yet developed hyperphosphatemia. In a study of patients with CKD who were not receiving dialysis (stage G3b or G4), had a mean baseline serum 
phosphate concentration of $1.36 \mathrm{mmol} / \mathrm{L}(4.2 \mathrm{mg} / \mathrm{dL})$, and were treated with 3 phosphate binders (sevelamer, lanthanum, or calcium acetate) versus matching placebo (29), there was a small decrease in serum phosphate concentrations and a $22 \%$ decrease in urinary phosphate excretion (suggesting adherence to therapy) in the active treatment group; no differences in changes in FGF23 levels were observed versus placebo. Contrary to expectations, progression of coronary and aortic calcification was observed with active phosphate binder treatment (primarily due to calcium acetate) but not with placebo.

This study was supported by another metabolic study in a small group of patients with CKD stage G3b or G4, in whom the addition of calcium carbonate (equivalent to three 500-mg doses of elemental calcium) to 3 daily meals containing $1 \mathrm{~g}$ of calcium and 1.5 $\mathrm{g}$ of phosphorus did not affect baseline neutral phosphate balance but caused a positive short-term calcium balance (30). Although this study did not meet the criteria for full evidence review, it may present a plausible and relevant safety signal.

Both studies examined patients with essentially normal phosphate concentrations at baseline $(29,30)$. Two conclusions are apparent: Normophosphatemia may not be an indication to start phosphate-lowering treatments, and not all phosphate binders are interchangeable. The recommendation was updated to clarify that phosphate-lowering therapies may only be indicated in the event of progressive or persistent hyperphosphatemia and not for prevention.

The metabolic study (30) supported results of an earlier study suggesting the potential harm of liberal calcium exposure in normophosphatemic adults with CKD stage G3b or G4 (38). The earlier study also was not eligible for full evidence review.

These results, together with uncertainties about phosphate-lowering therapy in patients with CKD who are not receiving dialysis and results of additional randomized controlled trials (RCTs) with hard end points $(29,44,45)$, prompted reevaluation of the 2009 recommendation with regard to calcium-based phosphate binders. The studies seemed to show either a potential for benefit or an absence of harm associated with calcium-free phosphate-binding agents compared with calcium-based agents for treatment of hyperphosphatemia.

The current evidence suggests that excess exposure to calcium may be harmful across all GFR categories of CKD. Despite the understandable desire to have numerical targets and limits, no explicit recommendation about a maximum dose of calcium-based binders was possible. Instead, phosphate-lowering treatment decisions should be individualized.

4.1.8: In patients with CKD G3a to G5D, we suggest limiting dietary phosphate intake in the treatment of hyperphosphatemia alone or in combination with other treatments. (Grade 2D recommendation) It is reasonable to consider phosphate source (e.g., animal, vegetable, additives) in making dietary recommendations. (Not graded)
There was no controversy about restricting dietary phosphate to decrease elevated phosphate levels, but the wording of the original statement was vague, especially in light of new evidence on different phosphate and phosphoprotein sources (processed vs. fresh food [46-49], vegetables vs. meat [15], and "hidden" sources $[49,50])$. Given that studies on various types of nutrition education have had mixed results for control of serum phosphate levels, the original recommendation on dietary phosphate restriction was amended to acknowledge that phosphate sources should be better substantiated and patient education should focus on best choices.

\section{UPDATED RECOMMENDATIONS RELATING TO Management of Serum PTH LeVels}

4.2.1: In patients with CKD G3a to G5 not on dialysis, the optimal PTH level is not known. However, we suggest that patients with levels of intact PTH progressively rising or persistently above the upper normal limit for the assay be evaluated for modifiable factors, including hyperphosphatemia, hypocalcemia, high phosphate intake, and vitamin D deficiency. (Grade 2C recommendation)

The pathogenesis of SHPT is complex and is driven by several factors, including vitamin D deficiency, hypocalcemia, and hyperphosphatemia. As kidney function decreases, the incidence and severity of SHPT increase, leading to abnormalities in bone mineralization and turnover.

Data from RCTs are insufficient to define an optimal PTH level for patients with CKD stage G3a to G5 or clinical end points of hospitalization, fracture, or death. Modest increases in PTH levels may represent an appropriate adaptive response to decreasing kidney function due to phosphaturic effects and increasing bone resistance to PTH (51). Therefore, the original recommendation was revised to reflect treatment based on trends in PTH level (highlighting levels "progressively rising or persistently above the upper normal limit") rather than a single elevated value.

The data highlighted an additional modifiable risk factor for SHPT: high phosphate intake. This revision acknowledges that excess phosphate intake does not always result in hyperphosphatemia, especially in early CKD, but high intake may promote SHPT.

4.2.2: In adult patients with CKD G3a to G5 not on dialysis, we suggest that calcitriol and vitamin $D$ analogues not be routinely used. (Grade $2 \mathrm{C}$ recommendation) It is reasonable to reserve the use of calcitriol and vitamin $D$ analogues for patients with CKD G4 to G5 with severe and progressive hyperparathyroidism. (Not graded)

Prevention and treatment of SHPT are important because imbalances in mineral metabolism are associated with CKD-MBD, and higher PTH levels are associated with increased morbidity and mortality in patients with CKD. Although the 2009 guideline summarized multiple studies showing the ability of calcitriol or vitamin D analogues to decrease PTH levels, there was a 
notable lack of trials demonstrating improvements in patient-centered outcomes. Recent RCTs of calcitriol or vitamin $D$ analogues have supplemented the evidence base.

A double-blind RCT (PRIMO [Paricalcitol Capsule Benefits in Renal Failure-Induced Cardiac Morbidity]) in patients with CKD stage G3a to G4, mild to moderate left ventricular hypertrophy (LVH), and PTH levels of 50 to $300 \mathrm{pg} / \mathrm{mL}$ compared paricalcitol with placebo to test the primary hypothesis that paricalcitol reduces left ventricular mass index (LVMI) over 48 weeks (52). The intention-to-treat analysis revealed that paricalcitol did not reduce LVMI and did not modify diastolic function. The mean serum calcium level increased by 0.08 $\mathrm{mmol} / \mathrm{L}(0.32 \mathrm{mg} / \mathrm{dL})$ in the paricalcitol group versus a decrease of $0.06 \mathrm{mmol} / \mathrm{L}(0.25 \mathrm{mg} / \mathrm{dL})$ in the placebo group. Episodes of hypercalcemia were more common in the paricalcitol group (22.6\%) than the placebo group (0.9\%).

In another double-blind RCT (OPERA [Oral Paricalcitol in Stage 3-5 Chronic Kidney Disease]), patients with CKD stage G3a to G5, LVH, and PTH levels of 55 $\mathrm{pg} / \mathrm{mL}$ or greater were randomly assigned to receive paricalcitol or placebo (53). The primary end point (change in LVMI over 52 weeks) and secondary outcomes (such as measures of systolic and diastolic function) did not differ between groups. The median changes in serum calcium level were $0.08 \mathrm{mmol} / \mathrm{L}(0.32$ $\mathrm{mg} / \mathrm{dL})$ and $0.01 \mathrm{mmol} / \mathrm{L}(0.04 \mathrm{mg} / \mathrm{dL})$ in the paricalcitol and placebo groups, respectively. Hypercalcemia (serum calcium level $>2.55 \mathrm{mmol} / \mathrm{L}$ [>10.2 $\mathrm{mg} / \mathrm{dL}]$ ) was observed in $43.3 \%$ and $3.3 \%$ of participants in the paricalcitol and placebo groups, respectively; $70 \%$ of hypercalcemic patients received concomitant calciumbased phosphate binders. Hypercalcemia could be corrected by stopping use of the binder without changing the paricalcitol dose.

The results from the PRIMO and OPERA studies were supported by recent meta-analyses $(54,55)$. The Work Group agreed that the risk-benefit ratio for treating moderate PTH elevations was no longer favorable. Therefore, use of calcitriol or vitamin D analogues should be reserved for severe and progressive SHPT.

4.2.4: In patients with CKD G5D requiring $\mathrm{PTH}$ lowering therapy, we suggest calcimimetics, calcitriol, or vitamin $D$ analogues, or a combination of calcimimetics with calcitriol or vitamin $D$ analogues. (Grade $2 B$ recommendation)

Use of PTH-lowering therapies in patients with CKD stage G5D was reappraised on the basis of new studies of cinacalcet and vitamin D analogues, with a focus on the EVOLVE trial (41). No new trials of calcitriol or vitamin $D$ analogues with patient-level end points were identified.

The EVOLVE trial evaluated the effect of cinacalcet versus placebo on patient-level outcomes in 3883 patients receiving hemodialysis, using a composite end point of all-cause mortality, nonfatal myocardial infarction, hospitalization for unstable angina, congestive heart failure, and peripheral vascular events (41). The unadjusted primary composite end point showed a statistically nonsignificant reduction (hazard ratio, 0.93; $P=0.112$ ) with cinacalcet, but analyses adjusted for imbalances in baseline characteristics showed that this reduction was nominal (hazard ratio, 0.88; $P=0.008$ ). Further, an interaction between treatment and age $(P=$ 0.04 ) led to speculation that cinacalcet may be effective predominantly in older patients receiving dialysis.

No consensus was reached about whether the EVOLVE data were sufficient to recommend cinacalcet as a first-line option for all patients with SHPT and CKD stage G5D who require PTH-lowering therapy. One opinion is that the primary end point of the EVOLVE trial was negative. The alternative opinion is that secondary analyses found effects on patient-level end points, whereas there are no positive data on mortality or patient-centered end points from trials of calcitriol or other vitamin D analogues.

Given the lack of consensus and the higher acquisition cost of cinacalcet, the revised recommendation for PTH-lowering therapy in patients with CKD stage G5D now lists all acceptable treatment options in alphabetical order. Treatment choice should be guided by individual considerations about concomitant therapies and the patient's current calcium and phosphate levels.

\section{UPDATED RECOMMENDATION RELATING TO Treatment of Bone Abnormalities With BisPHOSPHONATES AND OTHER OSTEOPOROSIS Medications}

4.3.3: In patients with CKD G3a to G5D with biochemical abnormalities of CKD-MBD and low BMD and/or fragility fractures, we suggest that treatment choices take into account the magnitude and reversibility of the biochemical abnormalities and the progression of CKD, with consideration of a bone biopsy. (Grade 2D recommendation)

This recommendation serves as a reminder that when treatment choices are considered, their adverse effects must also be taken into account (for example, antiresorptives exacerbate low bone turnover, and denosumab may induce significant hypocalcemia) and the risks of administering antiresorptives must be weighed against the accuracy of the diagnosis of the underlying bone phenotype.

\section{Discussion}

The process of updating the 2009 CKD-MBD guideline to accommodate data from new studies found that many of the original recommendations remain current. Overall, 15 recommendations were revised.

Prospective studies evaluating BMD testing in adults with CKD represent a substantial advance since the original CKD-MBD guideline was published. The data support use of DXA BMD testing if the results will 
affect future treatment. Because such testing does not distinguish among types of renal osteodystrophy, bone biopsy remains the diagnostic gold standard. For patients at high risk for fracture, facilities that lack the ability to perform bone biopsy or evaluate the results should not withhold antiresorptive therapy.

The interplay among biochemical variables (serum phosphate, calcium, and PTH) in patients with CKDMBD received considerable attention during the review of the current evidence. It is apparent that therapeutic maneuvers aimed at improving one variable often have unintended effects on others. Thus, treatment approaches for CKD-MBD should be based on serial assessments of these variables taken together.

Current evidence does not show benefit to maintaining normal serum phosphate levels in patients not receiving dialysis, and there are safety concerns associated with aggressive phosphate-lowering therapy. Thus, treatment should focus on patients with overt hyperphosphatemia. In the case of calcium, new evidence suggests that hypercalcemia may be harmful in all GFR categories of CKD, prompting the recommendation to avoid inappropriate calcium loading in adults whenever possible. Use of calcium-based phosphate binders should also be restricted in patients with hyperphosphatemia across the CKD spectrum.

The 2009 recommendations for treatment of SHPT were expanded to reflect that modest increases in PTH may represent an appropriate adaptive response to decreasing kidney function. The current recommendation is to treat patients with PTH values that are progressively increasing or persistently above the upper limit of normal and not to base treatment on a single elevated value. Treatment approaches for SHPT in patients not receiving dialysis should not include routine use of calcitriol or vitamin D analogues due to the increased risk for hypercalcemia. Calcimimetics, calcitriol, and vitamin D analogues are acceptable first-line options in patients receiving dialysis.

Despite the recent clinical trials discussed in the updated guideline, significant gaps remain in the knowledge base for treatment of CKD-MBD, as demonstrated by the relatively small number of recommendations updated in the 2017 guideline. Future research should address many of these gaps. For example, RCTs should be conducted to compare the ability of calcium-containing and calcium-free phosphate binders to promote bone accrual, as well as their effect on arterial calcification. Studies on dietary phosphate intake should compare phosphate sources (vegetable, meat, or "hidden" sources [such as food additives]). Prospective trials should use a benefit-risk-cost ratio to identify the most effective phosphate-lowering approach across all CKD GFR categories; such studies should include patient-centered and surrogate end points, including vascular calcification, FGF23 levels, and LVH. Multicenter studies examining patient-level outcomes are needed to determine the benefits and risks of treatment with calcitriol or vitamin $\mathrm{D}$ analogues in patients with CKD stage G3a to G5 and mild or severe SHPT. Placebo-controlled trials are also needed to compare calcimimetics with standard therapy in patients with CKD stage G5D and SHPT, with an emphasis on FGF23 reduction as a therapeutic end point.

From Klinikum Coburg, Coburg, Germany, and University of Split School of Medicine, Split, Croatia; Denver Nephrology, Denver, Colorado; University Hospitals Leuven, Leuven, Belgium; Tokai University School of Medicine, Isehara, Japan; Hennepin County Medical Center and University of Minnesota, Minneapolis, Minnesota; Eagle, Idaho; Indiana University School of Medicine and Roudebush Veterans Affairs Medical Center, Indianapolis, Indiana; Great Ormond Street Hospital for Children, NHS Foundation Trust, London, United Kingdom; University of Calgary, Calgary, Alberta, Canada; The Royal Melbourne Hospital, University of Melbourne, Melbourne, Victoria, Australia; VU University Medical Center Amsterdam, Amsterdam, the Netherlands; and Stanford University School of Medicine, Stanford, California.

Acknowledgment: The Work Group thanks Bertram L. Kasiske and the KDIGO Co-Chairs, David C. Wheeler and Wolfgang C. Winkelmayer, for their invaluable guidance. The Work Group is also grateful for the rigorous evidence review performed by Dr. Karen A. Robinson and her evidence review team members. Finally, the Work Group acknowledges all who provided feedback during the public review of the draft guideline.

Financial Support: This guideline is supported by KDIGO, and no funding is accepted for the development of specific guidelines.

Disclosures: Dr. Ketteler reports grants from Amgen and personal fees from Amgen, Fresenius Medical Care, Medice, Sanofi, and Vifor Fresenius Medical Care Renal Pharma outside the submitted work. Dr. Block reports personal fees from Amgen, Kirin Corporation, OPKO, Daiichi Sankyo, ONO Pharmaceutical, Keryx, and Ardelyx; nonfinancial support from Amgen, OPKO, ONO Pharmaceutical, and Keryx; grants from Keryx; and other support from Ardelyx outside the submitted work. Dr. Evenepoel reports grants from Amgen and personal fees from Amgen, Sanofi, and Vifor Fresenius Medical Care during the conduct of the study. Dr. Fukagawa reports grants from Kyowa Hakko Kirin and Bayer Japan and personal fees from Kyowa Hakko Kirin, Bayer Japan, ONO Pharmaceutical, and Torii Pharmaceutical outside the submitted work. Dr. Herzog reports grants from Amgen and Zoll and personal fees from AbbVie, FibroGen, Relypsa, Sanifit, and ZS Pharma outside the submitted work. Ms. McCann reports other support from Amgen, Sanofi, and Relypsa outside the submitted work. Dr. Moe reports grants from Chugai, the National Institutes of Health, and the U.S. Department of Veterans Affairs and personal fees from Sanofi Genzyme and Amgen outside the submitted work. Dr. Toussaint reports grants and nonfinancial support from Amgen, Shire, and Sanofi during the conduct of the study. Dr. Vervloet reports grants from Fresenius Medical Care, Vifor Pharma, and Amgen and personal fees from Fresenius Medical Care, Vifor Pharma, Otsuka, Baxter, and Amgen outside the submitted work. Authors not named here have disclosed no conflicts of interest. Disclosures can also be viewed at www.acponline.org/authors/icmje/ConflictOf InterestForms.do?msNum=M17-2640. All Work Group members are required to complete, sign, and submit a financial disclosure and attestation form showing all such relationships 
that might be perceived as or are actual conflicts of interest. All reported information is published in the Work Group members' Biographic and Disclosure section, which can be found in the full-text guideline update (http://kdigo.org/wp-content /uploads/2017/02/2017-KDIGO-CKD-MBD-GL-Update.pdf).

Requests for Single Reprints: Markus Ketteler, MD, Division of Nephrology, Klinikum Coburg GmbH, Ketschendorfer Street 33, 96450 Coburg, Germany (e-mail, markus.ketteler @klinikum-coburg.de); Mary B. Leonard, MD, MSCE, Stanford University School of Medicine, 300 Pasteur Drive, Room G-306, Stanford, CA 94305 (e-mail, leonard5@stanford.edu).

Current author addresses and author contributions are available at Annals.org.

\section{References}

1. Kidney Disease: Improving Global Outcomes (KDIGO) CKD-MBD Work Group. KDIGO clinical practice guideline for the diagnosis, evaluation, prevention, and treatment of chronic kidney disease-mineral and bone disorder (CKD-MBD). Kidney Int Suppl. 2009:S1-130. [PMID: 19644521] doi:10.1038/ki.2009.188

2. Ketteler M, Block GA, Evenepoel P, Fukagawa M, Herzog CA, McCann L, et al. KDIGO 2017 clinical practice guideline update for the diagnosis, evaluation, prevention, and treatment of CKD-MBD. Kidney Int Suppl. 2017;7(Suppl 1):1-59. doi:10.1016/j.kisu.2017.04 .001

3. Ketteler M, Elder GJ, Evenepoel P, Ix JH, Jamal SA, Lafage-Proust $\mathrm{MH}$, et al. Revisiting KDIGO clinical practice guideline on chronic kidney disease-mineral and bone disorder: a commentary from a Kidney Disease: Improving Global Outcomes Controversies Conference. Kidney Int. 2015;87:502-28. [PMID: 25651364] doi:10.1038/ki .2014 .425

4. Guyatt G, Oxman AD, Akl EA, Kunz R, Vist G, Brozek J, et al. GRADE guidelines: 1. Introduction-GRADE evidence profiles and summary of findings tables. J Clin Epidemiol. 2011;64:383-94. [PMID: 21195583] doi:10.1016/j.jclinepi.2010.04.026

5. limori S, Mori Y, Akita W, Kuyama T, Takada S, Asai T, et al. Diagnostic usefulness of bone mineral density and biochemical markers of bone turnover in predicting fracture in CKD stage 5D patients-a single-center cohort study. Nephrol Dial Transplant. 2012;27:345-51. [PMID: 21652550] doi:10.1093/ndt/gfr317

6. Naylor KL, Garg AX, Zou G, Langsetmo L, Leslie WD, Fraser LA, et al. Comparison of fracture risk prediction among individuals with reduced and normal kidney function. Clin J Am Soc Nephrol. 2015; 10:646-53. [PMID: 25655423] doi:10.2215/CJN.06040614

7. West SL, Lok CE, Langsetmo L, Cheung AM, Szabo E, Pearce D, et al. Bone mineral density predicts fractures in chronic kidney disease. J Bone Miner Res. 2015;30:913-9. [PMID: 25400209] doi:10 $.1002 / j b m r .2406$

8. Yenchek RH, Ix JH, Shlipak MG, Bauer DC, Rianon NJ, Kritchevsky $\mathrm{SB}$, et al; Health, Aging, and Body Composition Study. Bone mineral density and fracture risk in older individuals with CKD. Clin J Am Soc Nephrol. 2012;7:1130-6. [PMID: 22516286] doi:10.2215/CJN .12871211

9. Jamal SA, Ljunggren O, Stehman-Breen C, Cummings SR, McClung MR, Goemaere S, et al. Effects of denosumab on fracture and bone mineral density by level of kidney function. J Bone Miner Res. 2011;26:1829-35. [PMID: 21491487] doi:10.1002/jbmr.403

10. Toussaint ND, Lau KK, Strauss BJ, Polkinghorne KR, Kerr PG. Effect of alendronate on vascular calcification in CKD stages 3 and 4: a pilot randomized controlled trial. Am J Kidney Dis. 2010;56:57-68. [PMID: 20347511] doi:10.1053/j.ajkd.2009.12.039

11. Haghverdi F, Farbodara T, Mortaji S, Soltani P, Saidi N. Effect of raloxifene on parathyroid hormone in osteopenic and osteoporotic postmenopausal women with chronic kidney disease stage 5. Iran J Kidney Dis. 2014;8:461-6. [PMID: 25362221]
12. Moe S, Drüeke T, Cunningham J, Goodman W, Martin K, Olgaard K, et al; Kidney Disease: Improving Global Outcomes (KDIGO). Definition, evaluation, and classification of renal osteodystrophy: a position statement from Kidney Disease: Improving Global Outcomes (KDIGO). Kidney Int. 2006;69:1945-53. [PMID: 16641930] 13. Sprague SM, Bellorin-Font E, Jorgetti V, Carvalho AB, Malluche $\mathrm{HH}$, Ferreira $\mathrm{A}$, et al. Diagnostic accuracy of bone turnover markers and bone histology in patients with CKD treated by dialysis. Am J Kidney Dis. 2016;67:559-66. [PMID: 26321176] doi:10.1053/j.ajkd .2015 .06 .023

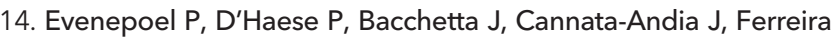
$A$, Haarhaus $M$, et al; ERA-EDTA Working Group on CKD-MBD. Bone biopsy practice patterns across Europe: the European Renal Osteodystrophy initiative-a position paper. Nephrol Dial Transplant. 2017;32:1608-13. [PMID: 28339949] doi:10.1093/ndt/gfw468

15. Moe SM, Zidehsarai MP, Chambers MA, Jackman LA, Radcliffe JS, Trevino LL, et al. Vegetarian compared with meat dietary protein source and phosphorus homeostasis in chronic kidney disease. Clin J Am Soc Nephrol. 2011;6:257-64. [PMID: 21183586] doi:10.2215 /CJN.05040610

16. Viaene L, Meijers B, Vanrenterghem Y, Evenepoel P. Daytime rhythm and treatment-related fluctuations of serum phosphorus concentration in dialysis patients. Am J Nephrol. 2012;35:242-8. [PMID: 22353750] doi:10.1159/000336308

17. Block GA, Kilpatrick RD, Lowe KA, Wang W, Danese MD. CKD mineral and bone disorder and risk of death and cardiovascular hospitalization in patients on hemodialysis. Clin J Am Soc Nephrol. 2013;8:2132-40. [PMID: 24052218] doi:10.2215/CJN.04260413

18. Chertow GM, Block GA, Correa-Rotter R, Drüeke TB, Floege J, Goodman WG, et al; EVOLVE Trial Investigators. Effect of cinacalcet on cardiovascular disease in patients undergoing dialysis. N Engl J Med. 2012;367:2482-94. [PMID: 23121374] doi:10.1056/NEJMoa 1205624

19. Chartsrisak K, Vipattawat K, Assanatham M, Nongnuch A, Ingsathit A, Domrongkitchaiporn S, et al. Mineral metabolism and outcomes in chronic kidney disease stage 2-4 patients. BMC Nephrol. 2013;14:14. [PMID: 23324569] doi:10.1186/1471-2369-14-14

20. Connolly GM, Cunningham R, McNamee PT, Young IS, Maxwell AP. Elevated serum phosphate predicts mortality in renal transplant recipients. Transplantation. 2009;87:1040-4. [PMID: 19352125] doi: 10.1097/TP.0b013e31819cd122

21. Eddington H, Hoefield R, Sinha S, Chrysochou C, Lane B, Foley $\mathrm{RN}$, et al. Serum phosphate and mortality in patients with chronic kidney disease. Clin J Am Soc Nephrol. 2010;5:2251-7. [PMID: 20688884] doi:10.2215/CJN.00810110

22. Fouque D, Roth $H$, Pelletier S, London GM, Hannedouche $T$, Jean $\mathrm{G}$, et al. Control of mineral metabolism and bone disease in haemodialysis patients: which optimal targets? Nephrol Dial Transplant. 2013;28:360-7. [PMID: 23136211] doi:10.1093/ndt/gfs404 23. Fukagawa M, Kido R, Komaba H, Onishi Y, Yamaguchi T, Hasegawa $\mathrm{T}$, et al. Abnormal mineral metabolism and mortality in hemodialysis patients with secondary hyperparathyroidism: evidence from marginal structural models used to adjust for time-dependent confounding. Am J Kidney Dis. 2014;63:979-87. [PMID: 24119541] doi: 10.1053/j.ajkd.2013.08.011

24. Lacson E Jr, Wang W, Hakim RM, Teng M, Lazarus JM. Associates of mortality and hospitalization in hemodialysis: potentially actionable laboratory variables and vascular access. Am J Kidney Dis. 2009;53:79-90. [PMID: 18930570] doi:10.1053/j.ajkd.2008.07.031

25. McGovern AP, de Lusignan S, van Vlymen J, Liyanage H, Tomson $\mathrm{CR}$, Gallagher $\mathrm{H}$, et al. Serum phosphate as a risk factor for cardiovascular events in people with and without chronic kidney disease: a large community based cohort study. PLoS One. 2013;8: e74996. [PMID: 24040373] doi:10.1371/journal.pone.0074996

26. Moore J, Tomson CR, Tessa Savage M, Borrows R, Ferro CJ. Serum phosphate and calcium concentrations are associated with reduced patient survival following kidney transplantation. Clin Transplant. 2011;25:406-16. [PMID: 20608946] doi:10.1111/j.1399-0012 .2010.01292.x 
27. Nakai S, Akiba T, Kazama J, Yokoyama K, Fukagawa M, Tominaga $Y$, et al; Patient Registration Committee of the Japanese Society for Dialysis Therapy, Tokyo, Japan. Effects of serum calcium, phosphorous, and intact parathyroid hormone levels on survival in chronic hemodialysis patients in Japan. Ther Apher Dial. 2008;12: 49-54. [PMID: 18257812] doi:10.1111/j.1744-9987.2007.00540.x 28. Tentori F, Blayney MJ, Albert JM, Gillespie BW, Kerr PG, Bommer J, et al. Mortality risk for dialysis patients with different levels of serum calcium, phosphorus, and PTH: the Dialysis Outcomes and Practice Patterns Study (DOPPS). Am J Kidney Dis. 2008;52:519-30. [PMID: 18514987] doi:10.1053/j.ajkd.2008.03.020

29. Block GA, Wheeler DC, Persky MS, Kestenbaum B, Ketteler M, Spiegel DM, et al. Effects of phosphate binders in moderate CKD. J Am Soc Nephrol. 2012;23:1407-15. [PMID: 22822075] doi:10.1681 /ASN.2012030223

30. Hill KM, Martin BR, Wastney ME, McCabe GP, Moe SM, Weaver $\mathrm{CM}$, et al. Oral calcium carbonate affects calcium but not phosphorus balance in stage 3-4 chronic kidney disease. Kidney Int. 2013;83: 959-66. [PMID: 23254903] doi:10.1038/ki.2012.403

31. Coen G, Pierantozzi A, Spizzichino D, Sardella D, Mantella D, Manni M, et al. Risk factors of one year increment of coronary calcifications and survival in hemodialysis patients. BMC Nephrol. 2010; 11:10. [PMID: 20565936] doi:10.1186/1471-2369-11-10

32. Fein PA, Asadi S, Singh P, Hartman W, Stuto S, Chattopadhyay J, et al. Relationship between alkaline phosphatase and all-cause mortality in peritoneal dialysis patients. Adv Perit Dial. 2013;29:61-3. [PMID: 24344494]

33. Floege J, Kim J, Ireland E, Chazot C, Drueke T, de Francisco A, et al; ARO Investigators. Serum iPTH, calcium and phosphate, and the risk of mortality in a European haemodialysis population. Nephrol Dial Transplant. 2011;26:1948-55. [PMID: 20466670] doi:10 $.1093 /$ ndt/gfq219

34. Markaki A, Kyriazis J, Stylianou K, Fragkiadakis GA, Perakis K, Margioris $\mathrm{AN}$, et al. The role of serum magnesium and calcium on the association between adiponectin levels and all-cause mortality in end-stage renal disease patients. PLoS One. 2012;7:e52350. [PMID: 23285003] doi:10.1371/journal.pone.0052350

35. Gallieni M, Caputo F, Filippini A, Gabella P, Giannattasio M, Stingone $A$, et al; ROCK-PD Study Investigators. Prevalence and progression of cardiovascular calcifications in peritoneal dialysis patients: a prospective study. Bone. 2012;51:332-7. [PMID: 22699014]

36. Nakano C, Hamano T, Fujii N, Matsui I, Tomida K, Mikami S, et al. Combined use of vitamin D status and FGF23 for risk stratification of renal outcome. Clin J Am Soc Nephrol. 2012;7:810-9. [PMID: 22362065] doi:10.2215/CJN.08680811

37. Raggi P, Bommer J, Chertow GM. Valvular calcification in hemodialysis patients randomized to calcium-based phosphorus binders or sevelamer. J Heart Valve Dis. 2004;13:134-41. [PMID: 14765851] 38. Spiegel DM, Brady K. Calcium balance in normal individuals and in patients with chronic kidney disease on low- and high-calcium diets. Kidney Int. 2012;81:1116-22. [PMID: 22297674] doi:10.1038/ ki.2011.490

39. Chonchol M, Locatelli F, Abboud HE, Charytan C, de Francisco $A L$, Jolly $S$, et al. A randomized, double-blind, placebo-controlled study to assess the efficacy and safety of cinacalcet $\mathrm{HCl}$ in participants with CKD not receiving dialysis. Am J Kidney Dis. 2009;53:197207. [PMID: 19110359] doi:10.1053/j.ajkd.2008.09.021

40. St Peter WL, Li Q, Liu J, Persky M, Nieman K, Arko C, et al. Cinacalcet use patterns and effect on laboratory values and other medications in a large dialysis organization, 2004 through 2006. Clin
J Am Soc Nephrol. 2009;4:354-60. [PMID: 19129318] doi:10.2215/ CJN.05241008

41. Moe SM, Chertow GM, Parfrey PS, Kubo Y, Block GA, CorreaRotter $\mathrm{R}$, et al; Evaluation of Cinacalcet $\mathrm{HCl}$ Therapy to Lower Cardiovascular Events (EVOLVE) Trial Investigators*. Cinacalcet, fibroblast growth factor-23, and cardiovascular disease in hemodialysis: the Evaluation of Cinacalcet $\mathrm{HCl}$ Therapy to Lower Cardiovascular Events (EVOLVE) trial. Circulation. 2015;132:27-39. [PMID: 26059012] doi:10.1161/CIRCULATIONAHA.114.013876

42. Ok E, Asci G, Bayraktaroglu S, Toz H, Ozkahya M, Yilmaz M, et al. Reduction of dialysate calcium level reduces progression of coronary artery calcification and improves low bone turnover in patients on hemodialysis. J Am Soc Nephrol. 2016;27:2475-86. [PMID: 26701977] doi:10.1681/ASN.2015030268

43. Spasovski G, Gelev S, Masin-Spasovska J, Selim G, Sikole A, Vanholder R. Improvement of bone and mineral parameters related to adynamic bone disease by diminishing dialysate calcium. Bone. 2007;41:698-703. [PMID: 17643363]

44. Di lorio B, Bellasi A, Russo D; INDEPENDENT Study Investigators. Mortality in kidney disease patients treated with phosphate binders: a randomized study. Clin J Am Soc Nephrol. 2012;7:48793. [PMID: 22241819] doi:10.2215/CJN.03820411

45. Di lorio B, Molony D, Bell C, Cucciniello E, Bellizzi V, Russo D, et al; INDEPENDENT Study Investigators. Sevelamer versus calcium carbonate in incident hemodialysis patients: results of an open-label 24-month randomized clinical trial. Am J Kidney Dis. 2013;62:771-8. [PMID: 23684755] doi:10.1053/j.ajkd.2013.03.023

46. Benini O, D'Alessandro C, Gianfaldoni D, Cupisti A. Extraphosphate load from food additives in commonly eaten foods: a real and insidious danger for renal patients. J Ren Nutr. 2011;21:303-8. [PMID: 21055967] doi:10.1053/j.jrn.2010.06.021

47. Sherman RA, Mehta O. Phosphorus and potassium content of enhanced meat and poultry products: implications for patients who receive dialysis. Clin J Am Soc Nephrol. 2009;4:1370-3. [PMID: 19628683] doi:10.2215/CJN.02830409

48. Calvo MS. Dietary considerations to prevent loss of bone and renal function. Nutrition. 2000;16:564-6. [PMID: 10906557]

49. Anderson JJB, Sell ML, Garner S, Calvo MS. Phosphorus. In: Bowman BA, Russell RM, eds. Present Knowledge in Nutrition. 8th ed. Washington, DC: ILSI Pr; 2001:281-91.

50. Sherman iRA, Ravella S, Kapoian T. The phosphate content of prescription medication: a new consideration. Ther Innov Regul Sci. 2015;49:886-9.

51. Evenepoel P, Bover J, Ureña Torres P. Parathyroid hormone metabolism and signaling in health and chronic kidney disease. Kidney Int. 2016;90:1184-90. [PMID: 27653840] doi:10.1016/j.kint.2016.06 .041

52. Thadhani R, Appelbaum E, Pritchett $Y$, Chang Y, Wenger J, Tamez $\mathrm{H}$, et al. Vitamin $\mathrm{D}$ therapy and cardiac structure and function in patients with chronic kidney disease: the PRIMO randomized controlled trial. JAMA. 2012;307:674-84. [PMID: 22337679] doi:10 .1001/jama.2012.120

53. Wang AY, Fang F, Chan J, Wen YY, Qing S, Chan IH, et al. Effect of paricalcitol on left ventricular mass and function in CKD-the OPERA trial. J Am Soc Nephrol. 2014;25:175-86. [PMID: 24052631] doi:10.1681/ASN.2013010103

54. Li XH, Feng L, Yang ZH, Liao YH. The effect of active vitamin D on cardiovascular outcomes in predialysis chronic kidney diseases: a systematic review and meta-analysis. Nephrology (Carlton). 2015;20: 706-14. [PMID: 25963841] doi:10.1111/nep.12505.

55. Xu L, Wan X, Huang Z, Zeng F, Wei G, Fang D, et al. Impact of vitamin $\mathrm{D}$ on chronic kidney diseases in non-dialysis patients: a metaanalysis of randomized controlled trials. PLoS One. 2013;8:e61387. [PMID: 23626678] doi:10.1371/journal.pone.0061387 
Current Author Addresses: Dr. Ketteler: Division of Nephrology, Klinikum Coburg GmbH, Ketschendorfer Street 33, 96450 Coburg, Germany.

Dr. Block: Denver Nephrology, 130 Rampart Way, Suite 175, Denver, CO 80230.

Dr. Evenepoel: University Hospitals Leuven, Herestraat 49, Leuven 3000, Belgium.

Dr. Fukagawa: Division of Nephrology, Endocrinology and Metabolism, Tokai University School of Medicine, 143 Shimokasuya, Isehara 2591193, Japan.

Dr. Herzog: Hennepin County Medical Center/University of Minnesota, 914 South 8th Street, Suite S-4.100, Minneapolis, MN 55404

Ms. McCann: 1406 North Foresto Bello Way, Eagle, ID 83616. Dr. Moe: Indiana University, 950 West Walnut Street, R2-202, Indianapolis, IN 46202.

Dr. Shroff: Great Ormond Street Hospital for Children, NHS Foundation Trust, Great Ormond Street, London WC1N 3JH, United Kingdom.

Dr. Tonelli: University of Calgary, TRW 7th Floor, 3280 Hospital Drive Northwest, Calgary, Alberta T6G 2G3, Canada.

Dr. Toussaint: The Royal Melbourne Hospital, University of Melbourne, Melbourne, Victoria 3050, Australia.

Dr. Vervloet: VU University Medical Center, De Boelelaan 1117, 1081 HV Amsterdam, the Netherlands.

Dr. Leonard: Stanford University School of Medicine, 300 Pasteur Drive, Room G-306, Stanford, CA 94305.
Author Contributions: Conception and design: M. Ketteler, G.A. Block, M. Fukagawa, S.M. Moe, R. Shroff, N.D. Toussaint, M.G. Vervloet.

Analysis and interpretation of the data: M. Ketteler, G.A Block, P. Evenepoel, L. McCann, S.M. Moe, R. Shroff, M.A. Tonelli, N.D. Toussaint, M.G. Vervloet, M.B. Leonard.

Drafting of the article: M. Ketteler, G.A. Block, L. McCann, R. Shroff, N.D. Toussaint, M.G. Vervloet.

Critical revision of the article for important intellectual content: M. Ketteler, G.A. Block, P. Evenepoel, C.A. Herzog, L. McCann, S.M. Moe, R. Shroff, M.A. Tonelli, N.D. Toussaint, M.G. Vervloet.

Final approval of the article: M. Ketteler, G.A. Block, P. Evenepoel, M. Fukagawa, C.A. Herzog, L. McCann, S.M. Moe, R. Shroff, M.A. Tonelli, N.D. Toussaint, M.G. Vervloet, M.B. Leonard.

Administrative, technical, or logistic support: M.B. Leonard. Collection and assembly of data: M. Ketteler, G.A. Block, P. Evenepoel, M. Fukagawa, L. McCann, N.D. Toussaint, M.G. Vervloet.

\section{Appendix Figure. Prognosis of CKD, by categories of GFR and albuminuria.}

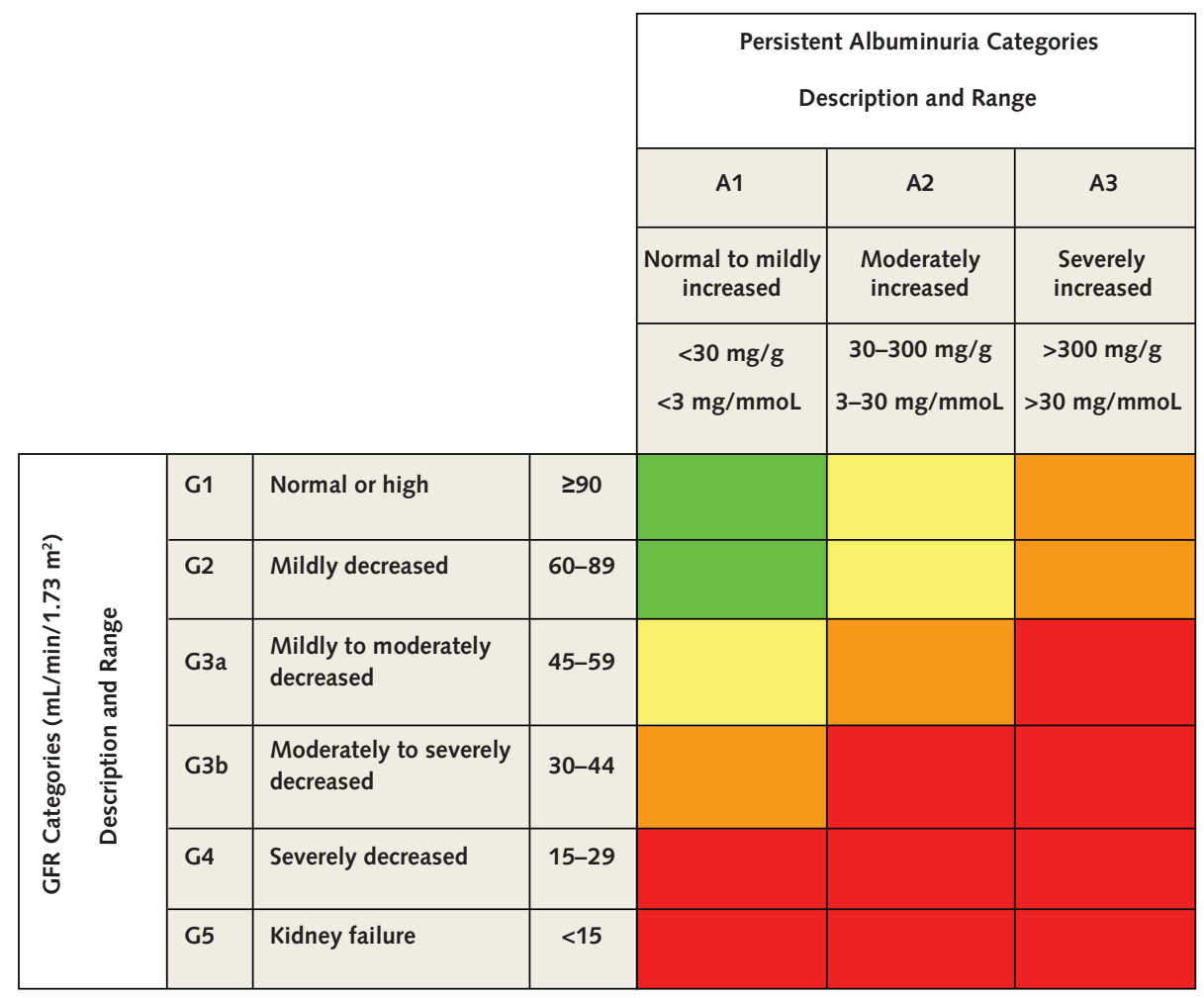

CKD is defined as abnormalities of kidney structure or function that are present for $>3$ mo and have health implications. CKD is classified on the basis of cause, GFR category (G1 to G5), and albuminuria category (A1 to A3). Green means low risk (no CKD if no other markers of kidney disease), yellow means moderately increased risk, orange means high risk, and red means very high risk. The suffix " $D$ " denotes dialysis (e.g., CKD G5D refers to a patient with CKD stage G5 who is receiving dialysis). (Reproduced with permission of Kidney Disease: Improving Global Outcomes.) CKD = chronic kidney disease; GFR = glomerular filtration rate. 


\begin{tabular}{|c|c|c|c|}
\hline \multirow[t]{2}{*}{ Grade } & \multicolumn{3}{|c|}{ Implications } \\
\hline & Patients & Clinicians & Policy \\
\hline Level 1: "We recommend" & $\begin{array}{l}\text { Most people in your situation would want } \\
\text { the recommended course of action, } \\
\text { and only a small proportion would not. }\end{array}$ & $\begin{array}{l}\text { Most patients should receive the } \\
\text { recommended course of action. }\end{array}$ & $\begin{array}{l}\text { The recommendation can be } \\
\text { evaluated as a candidate for } \\
\text { developing a policy or a } \\
\text { performance measure. }\end{array}$ \\
\hline Level 2: "We suggest" & $\begin{array}{l}\text { The majority of people in your situation } \\
\text { would want the recommended course } \\
\text { of action, but many would not. }\end{array}$ & $\begin{array}{l}\text { Different choices will be appropriate for } \\
\text { different patients. Each patient needs } \\
\text { help to arrive at a management } \\
\text { decision consistent with her or his } \\
\text { values and preferences. }\end{array}$ & $\begin{array}{l}\text { The recommendation is likely to } \\
\text { require debate and involvement } \\
\text { of stakeholders before policy } \\
\text { can be determined. }\end{array}$ \\
\hline
\end{tabular}

GRADE $=$ Grading of Recommendations Assessment, Development and Evaluation .

* The additional category "not graded" is typically used to provide guidance based on common sense or when the topic does not allow adequate application of evidence. The most common examples include recommendations regarding monitoring intervals, counseling, and referral to other clinical specialists. The ungraded recommendations are generally written as simple declarative statements but are not meant to be interpreted as being stronger recommendations than level 1 or 2 recommendations.

\begin{tabular}{lll}
\hline Appendix Table 2. GRADE Criteria Used for Grading the Overall Quality of Evidence \\
\hline Grade & $\begin{array}{l}\text { Quality of } \\
\text { Evidence }\end{array}$ & Meaning \\
\hline A & High & We are confident that the true effect lies close to that of the estimate of the effect. \\
B & Moderate & The true effect is likely to be close to the estimate of the effect, but there is a possibility that it is substantially different. \\
C & Low & The true effect may be substantially different from the estimate of the effect. \\
D & Very low & The estimate of the effect is very uncertain and often will be far from the truth. \\
\hline
\end{tabular}

GRADE = Grading of Recommendations Assessment, Development and Evaluation . 


\begin{tabular}{|c|c|c|c|}
\hline $\begin{array}{l}2009 \text { Recommendation } \\
\text { Number }\end{array}$ & Research Question & Key Outcomes & Additional Outcomes \\
\hline 4.3.4 & $\begin{array}{l}\text { In patients with CKD G4 to G5D, what is the effect on } \\
\text { bone quality of bisphosphonates, teriparatide, } \\
\text { denosumab, and raloxifene? }\end{array}$ & $\begin{array}{l}\text { TMV (as measured by bone biopsy) } \\
\text { BMD/bone mineral content } \\
\text { Fracture }\end{array}$ & - \\
\hline 3.2 .2 & $\begin{array}{l}\text { In patients with CKD G3a to G5D, how well do BMD } \\
\text { results predict fractures? } \\
\text { In patients with CKD G3a to G5D, how well do BMD } \\
\text { results predict renal osteodystrophy? }\end{array}$ & $\begin{array}{l}\text { Fracture } \\
\text { TMV }\end{array}$ & - \\
\hline \multicolumn{4}{|l|}{ Calcium and phosphate } \\
\hline 4.1.1 & $\begin{array}{l}\text { In patients with CKD G3a to G5 or G5D, what is the } \\
\text { evidence for benefit or harm in maintaining serum } \\
\text { phosphate in the normal range compared with } \\
\text { other targets of serum phosphate in terms of } \\
\text { biochemical outcomes, other surrogate outcomes, } \\
\text { and patient-centered outcomes? }\end{array}$ & $\begin{array}{l}\text { Mortality } \\
\text { GFR decline } \\
\text { Cardiovascular and } \\
\quad \text { cerebrovascular events }\end{array}$ & $\begin{array}{l}\text { Phosphate } \\
\text { Bone histology, BMD } \\
\text { Vascular and valvular calcification } \\
\quad \text { imaging } \\
\text { Hospitalizations } \\
\text { Quality of life } \\
\text { Kidney or kidney graft failure } \\
\text { Fracture } \\
\text { Parathyroidectomy } \\
\text { Clinical adverse events } \\
\text { Growth, skeletal deformities, bone } \\
\text { accrual } \\
\text { Calciphylaxis/CUA }\end{array}$ \\
\hline 4.1 .3 & $\begin{array}{l}\text { In patients with CKD G5D, what is the evidence for } \\
\text { benefit or harm in using a dialysate calcium } \\
\text { concentration between } 1.25 \text { and } 1.50 \mathrm{mmol} / \mathrm{L} \\
(2.5 \text { and } 3.0 \mathrm{mEq} / \mathrm{L}) \text { compared with other concen- } \\
\text { trations of dialysate calcium in terms of biochemical } \\
\text { outcomes, other surrogate outcomes, and } \\
\text { patient-centered outcomes? }\end{array}$ & $\begin{array}{l}\text { Mortality } \\
\text { Cardiovascular and } \\
\quad \text { cerebrovascular events }\end{array}$ & $\begin{array}{l}\text { Calcium } \\
\text { Bone histology, BMD } \\
\text { Vascular and valvular calcification } \\
\quad \text { imaging } \\
\text { Measures of GFR } \\
\text { Hospitalizations } \\
\text { Quality of life } \\
\text { Kidney or kidney graft failure } \\
\text { Fracture } \\
\text { Parathyroidectomy } \\
\text { Clinical adverse events } \\
\text { Growth, skeletal deformities, bone } \\
\quad \text { accrual } \\
\text { Calciphylaxis/CUA }\end{array}$ \\
\hline 4.1 .4 & $\begin{array}{l}\text { In patients with CKD G3a to G5 or G5D with } \\
\text { hyperphosphatemia, what is the evidence for } \\
\text { benefit or harm in using calcium-containing } \\
\text { phosphate-binding agents to treat hyperphosp- } \\
\text { hatemia compared with calcium-free phosphate- } \\
\text { binding agents in terms of biochemical outcomes, } \\
\text { other surrogate outcomes, and patient-centered } \\
\text { outcomes? }\end{array}$ & $\begin{array}{l}\text { Mortality } \\
\text { Cardiovascular and } \\
\quad \text { cerebrovascular events }\end{array}$ & $\begin{array}{l}\text { Phosphate } \\
\text { Bone histology, BMD } \\
\text { Vascular and valvular calcification } \\
\text { imaging } \\
\text { Measures of GFR } \\
\text { Hospitalizations } \\
\text { Quality of life } \\
\text { Kidney or kidney graft failure } \\
\text { Fracture } \\
\text { Parathyroidectomy } \\
\text { Clinical adverse events } \\
\text { Growth, skeletal deformities, bone } \\
\text { accrual } \\
\text { Calciphylaxis/CUA }\end{array}$ \\
\hline
\end{tabular}

Continued on following page 


\begin{tabular}{|c|c|}
\hline \multicolumn{2}{|c|}{ Appendix Table 3-Continued } \\
\hline $\begin{array}{l}2009 \text { Recommendation } \\
\text { Number }\end{array}$ & Research Question \\
\hline 4.1 .7 & $\begin{array}{l}\text { In patients with CKD G3a to G5D with } \\
\text { hyperphosphatemia, what is the evidence for } \\
\text { benefit or harm in limiting dietary phosphate } \\
\text { intake compared with a standard diet in terms of } \\
\text { biochemical outcomes, other surrogate outcomes, } \\
\text { and patient-centered outcomes? }\end{array}$ \\
\hline
\end{tabular}

Vitamin D and PTH

4.2.1

4.2 .2
In patients with CKD G3a to $\mathrm{G} 5$ not receiving dialysis with levels of intact PTH above the upper normal limit, what is the evidence for benefit or harm in reducing dietary phosphate intake or treating with phosphate-binding agents, calcium supplements, or native vitamin $D$ in terms of biochemical outcomes, other surrogate outcomes, and patient-centered outcomes?
Key Outcomes

\section{Mortality}

Cardiovascular and cerebrovascular events

Vascular and valvular calcification

\section{Mortality}

Cardiovascular and cerebrovascular events

GFR decrease
Additional Outcomes

Phosphate

Bone histology, BMD

Measures of GFR

Hospitalizations

Quality of life

Kidney or kidney graft failure

Fracture

Parathyroidectomy

Clinical adverse events

Growth, skeletal deformities, bone accrual

Calciphylaxis/CUA

\section{Calcium}

Phosphate

PTH

25- $(\mathrm{OH}) \mathrm{D}$

1,25- $(\mathrm{OH})_{2} \mathrm{D}$

Alkaline phosphatases

Bone-specific alkaline phosphatase

Bicarbonate

FGF23

Bone histology, BMD

Vascular and valvular calcification imaging

Measures of GFR

Hospitalizations

Quality of life

Kidney or kidney graft failure

Fracture

Parathyroidectomy

Clinical adverse events

Growth, skeletal deformities, bone accrual

Calciphylaxis/CUA

Calcium

Phosphate

PTH

25- $(\mathrm{OH}) \mathrm{D}$

$1,25-(\mathrm{OH})_{2} \mathrm{D}$

Alkaline phosphatase

Bone-specific alkaline phosphatase

Bicarbonate

FGF23

Bone histology, BMD

Vascular and valvular calcification imaging

Measures of GFR

Hospitalizations

Quality of life

Kidney or kidney graft failure

Fracture

Parathyroidectomy

Clinical adverse events

Growth, skeletal deformities, bone accrual

Calciphylaxis/CUA

Continued on following page 


\begin{tabular}{|c|c|c|c|}
\hline \multicolumn{4}{|c|}{ Appendix Table 3-Continued } \\
\hline $\begin{array}{l}2009 \text { Recommendation } \\
\text { Number }\end{array}$ & Research Question & Key Outcomes & Additional Outcomes \\
\hline
\end{tabular}

1,25-(OH) $2 \mathrm{D}=1,25$-dihydroxyvitamin $\mathrm{D} ; 25-(\mathrm{OH}) \mathrm{D}=25$-hydroxyvitamin $\mathrm{D} ; \mathrm{BMD}=$ bone mineral density; $\mathrm{CKD}=$ chronic kidney disease; $\mathrm{CUA}=$ calcific uremic arteriolopathy; FGF23 = fibroblast growth factor 23; GFR = glomerular filtration rate; LVH = left ventricular hypertrophy; PTH = parathyroid hormone; TMV = bone turnover mineralization volume.

Downloaded From: http://annals.org/ by a Ku Leuven- Univ Library User on 02/23/2018 